P53

\section{A stabilizing influence}

A newly identified interaction between the ribosomal protein $\mathrm{L} 11$ and a negative regulator of $\mathrm{p} 53, \mathrm{HDM} 2$, can lead to the stabilization and activation of p53 under conditions of altered ribosome biogenesis. This finding, as reported by Karen Vousden and colleagues in Cancer Cell, is thought to represent a new stress-induced pathway for p53 stabilization.

Using an HDM2 fragment as bait, Vousden and co-workers identified L11 in a yeast twohybrid screen. The interaction between L11 and HDM2 was enhanced in response to actinomycin-D treatment, which inhibits RNA polymerase I and leads to changes in ribosomal biogenesis.

When overexpressed, L11 stabilized both p53 and HDM2. The interaction of L11 with HDM2, which has E3 ligase activity, inhibited the ability of HDM2 to target both p 53 and

\section{When exercise is deadly}

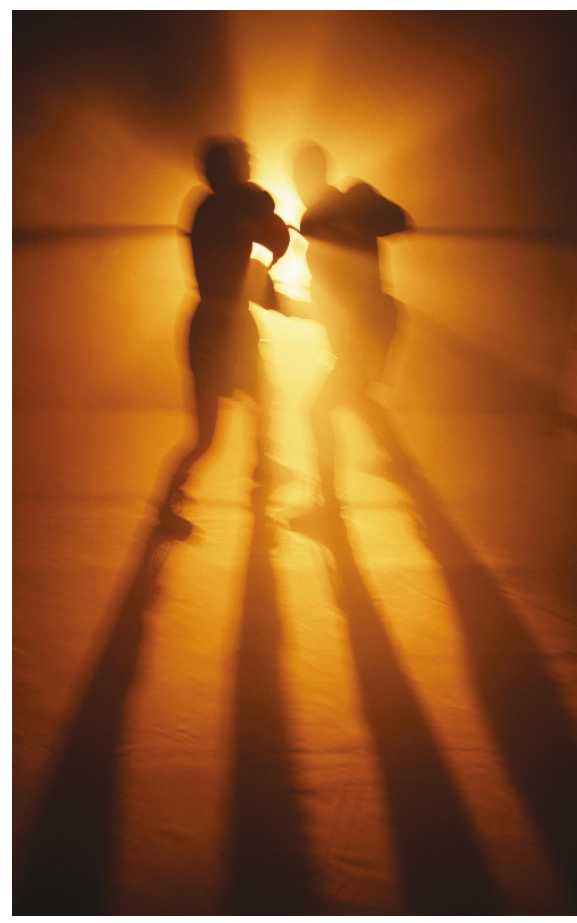

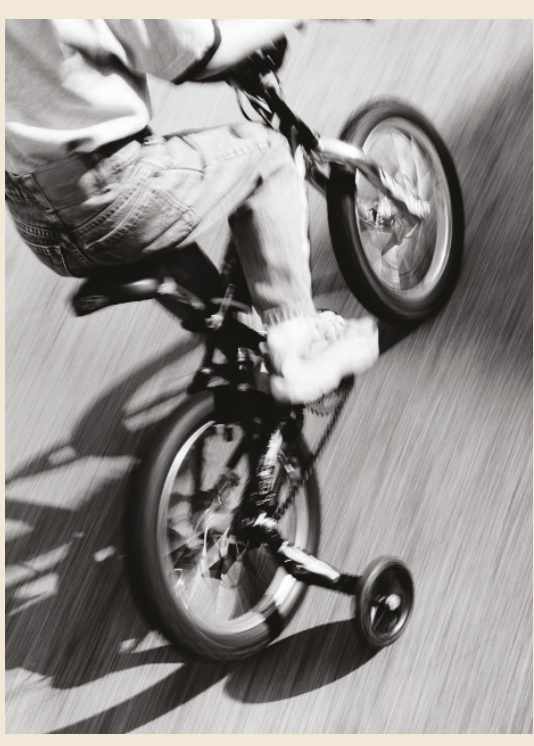

itself for ubiquitylation and degradation. Importantly, L11 expression had no effect on the stability of a p53 mutant that lacked the HDM2-binding site, which indicates that L11's main activity is to inhibit HDM2mediated p53 degradation. This function is similar to that of a small protein known as $\mathrm{ARF}$, and the authors suggest that there might be other HDM2-binding proteins that regulate $\mathrm{p} 53$ activity in response to different stress signals.

ARF-null cells that were transfected with L11 arrested in G1 - the same result that is seen in cells transfected with ARF. To show that this cell-cycle arrest was the result of $\mathrm{p} 53$ activation, the authors co-expressed papillomavirus protein $\mathrm{E} 6$, which targets $\mathrm{p} 53$ for degradation. Indeed, E6-mediated inhibition of $\mathrm{p} 53$ caused a decrease in G1 arrest.

The authors suggest that this new mechanism "...might play a role in the response to abnormalities in ribosomal biogenesis induced by abnormal metabolic conditions that could occur during tumor development". Whether $\mathrm{L} 11$ has a role in stabilizing p 53 in response to other stress signals remains an open question for now. Other ribosomal proteins that bind HDM2 might function in the same way.

\section{Arianne Heinrichs}

\section{(4) References and links}

ORIGINAL RESEARCH PAPER Lohrum, M. A. E. et al. Regulation of HDM2 activity by the ribosomal protein L11. Cancer Cell 3, 577-587 (2003)

FURTHER READING Marechal, V. et al. The ribosomal L5 protein is associated with $\mathrm{mdm}-2$ and $\mathrm{mdm}-2-\mathrm{p} 53$ complexes. Mol. Cell. Biol. 14, 714-720 (1994)
We all know that exercise is good for us, but there are times when it can be deadly. Patients with catecholaminergic polymorphic ventricular tachycardia (CPVT) experience exercise-induced arrhythmias, which can lead to sudden cardiac death in structurally normal hearts. But what triggers these fatal arrhythmias? In Cell, Marks and colleagues now report that the problem might be a 'leaky' $\mathrm{Ca}^{2+}$-release channel (the tetrameric ryanodine receptor, RyR2), which is mutated in CPVT patients.

During exercise, increased cyclic AMP levels in cardiac muscle activate cAMP-dependent protein kinase A (PKA). PKA then phosphorylates RyR2, which is on the sarcoplasmic reticulum, and this results in $\mathrm{Ca}^{2+}$ release and muscle contraction. The FK506-binding protein (FKBP12.6) - which binds non-phosphorylated RyR2 (one FKBP12.6 per RyR2 monomer) — stabilizes RyR2 to prevent $\mathrm{Ca}^{2+}$ release during the resting phase of the cardiac cycle (diastole).

The authors first examined FKBP12.6 $6^{-1}$ mice, and found that their hearts were structurally normal and that, at rest, these mice had no arrhythmias. However, under exercise conditions, FKBP $12.6^{-/-}$mice consistently had ventricular arrhythmias similar to those seen in CPVT patients.

Next, Marks and co-workers showed that, although RyR2 was PKA phosphorylated to the same level in FKBP12.6 $6^{+/+}$and FKBP12.6 $6^{-/-}$mice during exercise, RyR2 channels were more likely to be open in FKBP12.6 $6^{-/-}$mice. In fact, RyR2 channels in FKBP12.6 $6^{-/-}$mice are likely to be open during diastole, when they should be preventing $\mathrm{Ca}^{2+}$ release.

So, could this defective $\mathrm{Ca}^{2+}$ release be occurring in CPVT patients? The authors studied RyR2 channels containing CPVT-causing mutations, and found that, under rest conditions, these channels had wild-type properties. However, under exercise conditions, when the mutant channels were PKA phosphorylated, they were more likely to be open than wild-type channels. Furthermore, compared with wild-type channels, the mutant channels had a significantly decreased affinity for FKBP12.6.

Finally, Marks and colleagues showed that a mutant form of FKBP12.6, which can bind PKAphosphorylated RyR2, could restore the normal function of a CPVT-mutant RyR2 channel and of RyR2 channels in FKBP12.6 $6^{-/-}$mouse hearts. So, this work showed that, although a partial depletion of FKBP12.6 from phosphorylated RyR2 needs to occur to allow increased muscle contraction during exercise, too little FKBP12.6 in the RyR2 complex - in FKBP12.6 $6^{-1-}$ mice or CPVT patients - results in leaky RyR2 channels that can trigger fatal cardiac arryhthmias.

Rachel Smallridge

(2) References and links

ORIGINAL RESEARCH PAPER Wehrens, X. H. T. et al.

FKBP12.6 deficiency and defective calcium release channel

(ryanodine receptor) function linked to exercise-induced sudden cardiac death. Cell 113, 829-840 (2003) 